\title{
Pesquisas envolvendo instrumentos históricos matemáticos e a interface entre história e ensino: uma visão dos trabalhos desenvolvidos no GPEHM
}

Researches involving historical mathematical instruments and the interface between history and teaching: a vision of the works developed in GPEHM

\author{
Suziê Maria Albuquerque \\ Francisco Wagner Soares Oliveira \\ Eugeniano Brito Martins \\ Ana Carolina Costa Pereira
}

\section{Resumo}

O presente artigo aborda discussões sobre o uso de instrumentos históricos matemáticos e sua implicação na construção da interface entre a história e o ensino de matemática, tendo como objetivo elencar elementos dessa construção por meio do levantamento dos trabalhos publicados por membros do GPEHM no período de 2016 a 2018, tendo em vista que a partir de 2016 este grupo iniciou suas produções com foco na construção de interface com viés historiográfico atualizado. Nesse universo, foram selecionados os trabalhos que abordam o uso dos instrumentos matemáticos, com aporte de textos históricos originais. Para fins de análise foram estabelecidas categorias de classificação para as produções: ensaios teóricos, instrumentos matemáticos usados em cálculo numérico ou de medidas e instrumentos náuticos ou astronômicos. A partir disso foram elencados os elementos da construção de interface contida e a historiografia adotada, indicando que em nenhum dos trabalhos consta todo o processo de interface, mas mostra que o GPEHM tem realizado experiências nesse sentido no sentido de consolidar propostas que aproximam a história da matemática da prática didática e pedagógica.

Palavras-chave: Interface. História da Matemática. Ensino.

\section{Introdução}

A Educação Matemática desponta como campo de pesquisa, em que seu objeto de estudo diz respeito às relações e determinações entre ensino, aprendizagem e conhecimento matemático, de acordo com Lorenzato e Fiorentini (2001), indica-se amplo alcance no contexto escolar, desde a concepção, planejamento e formação didático e pedagógica, até as questões do cotidiano, da aplicação de atividades e avaliação da aprendizagem. 
Kilpatrick (1998) menciona que os estudos gerais relacionados ao ensino e aprendizagem da matemática têm dado espaço a investigações com foco a conhecimentos matemáticos específicos, requerendo recursos diversificados que favoreçam a compreensão destes conceitos de forma contextualizada.

Assim, a história "pode evidenciar que a Matemática não se limita a um sistema de regras e verdades rígidas, mas é algo humano e envolvente" (BARONI, TEIXEIRA e NOBRE, 2004, p. 167), motivando a introdução de novos conceitos por parte dos professores, desenvolvendo novas formas de observar e ensinar a matemática.

Saito (2015, p. 31) considera que a "história da matemática é o estudo das formas de elaboração, transformação e transmissão de conhecimento sobre as matemáticas, a natureza, as técnicas e as sociedades em diferentes épocas e culturas", distinguindo-a da educação matemática por terem objetos de investigação distintos. No entanto, a história revela o processo de construção do conhecimento matemático implicando no ensino e na aprendizagem destes.

Faz-se relevante discutir a interação entre essas duas grandes áreas de conhecimento, tendo em vista que estudos na articulação entre a história e o ensino de matemática (SAITO \& DIAS, 2013; SAITO, 2015) conquistaram espaço no meio acadêmico, diante do potencial integrador dessas duas áreas em função da intervenção didática e pedagógica nos diversos níveis e modalidades de educacionais.

No entanto, o trabalho com a história no ensino tem requerido a adoção de uma perspectiva historiográfica atualizada que, "[...] procura privilegiar não só o conteúdo matemático em si, mas também os documentos que versam a seu respeito." (SAITO, 2015, p. 26-27). Ou seja, a partir de documentos escritos ou outros vestígios históricos, como os instrumentos matemáticos, emerge todo o contexto de elaboração do conhecimento matemático, superando o conteúdo e, ao mesmo tempo, revelando seus elementos constitutivos.

Nesse sentido, despontam estudos que partem da construção e uso de instrumentos antigos, conduzindo à discussão de suas potencialidades didáticas 
para o ensino, como cita Pereira (2016) ao listar os trabalhos publicados no XI Seminário Nacional de História da Ciência e da Tecnologia (XI SNHCT) que lançam olhar sobre instrumentos matemáticos. Dos sete trabalhos mencionados pela autora, cinco foram de autoria de integrantes do Grupo de Pesquisas em Educação e História da Matemática (GPEHM), indicando o interesse e protagonismo deste coletivo com relação à temática.

O GPEHM foi escolhido como locus de investigação, pois é um dos grupos que estabelece parceria de trabalho com o HEEMa (História e Epistemologia na Educação Matemática), grupo da PUC-SP que desenvolve estudos sobre instrumentos matemáticos e a construção de interface entre a história e o ensino numa perspectiva historiográfica atualizada. Além disso, é o pioneiro no Estado do Ceará em estudos nesse sentido.

Diante do exposto, este trabalho se direciona ao ensino, partindo das necessidades implicadas no cenário da Educação Matemática e objetiva elencar a construção de interfaces entre a história e o ensino por meio da discussão de estudos que foram publicados por integrantes do GPEHM que versam sobre essa articulação.

Para tanto, foi realizada uma pesquisa qualitativa, de cunho bibliográfico na qual foram destacados os elementos dos estudos deste grupo de pesquisadores que discutem o uso de instrumentos matemáticos no Ensino.

\section{A interface entre história e ensino de matemática: discutindo ideias}

A história da matemática é comumente abordada em sala de aula, passando a ideia de continuidade no desenvolvimento dos conteúdos ensinados, essa é uma forma tradicional de compreender a história, quando o correto seria: "partir do passado em direção ao presente na medida que é a partir de um acontecimento do passado que se deve entender o presente, e não ao contrário" (SAITO, 2015, p. 27). 
Esta forma de escrever a história, conhecida como historiografia atualizada, permite compreender a matemática na visão, nas definições e nas concepções da época histórica em estudo. Compreendendo a rede de conhecimentos matemáticos mobilizados no passado para a construção de determinados conceitos em seu contexto de elaboração, de forma que estes possam dialogar com o ensino atual. Aproximando assim a história e o ensino da matemática, pois:

A articulação entre história e ensino, portanto, requer que consideremos não só aspectos epistemológicos e metodológicos ligados à história da matemática, mas também, à educação matemática. Neste sentido, o diálogo entre historiadores da matemática e educadores da matemática faz-se essencial. (SAITO, 2016, p. 256)

A realização deste diálogo com a intenção de articular as duas áreas do conhecimento escolhidas, é que possibilita a construção das interfaces, entendidas como "[...] um conjunto de ações e produções que promova a reflexão sobre o processo histórico da construção do conhecimento matemático para elaborar atividades didáticas que busquem articular história e ensino de matemática." (SAITO \& DIAS, 2013, p. 92).

Desta forma, a interface é o resultado de uma reflexão sobre o processo como se deu a criação ou mobilização dos conceitos matemáticos em um momento histórico, acrescido de ações e produções que tragam estes conceitos para auxiliar o ensino da matemática, obtidas por intermédio de diálogos entre o educador e o historiador da matemática, existindo duas razões para a sua necessidade:

1) para aproximar o educador matemático dos recentes desenvolvimentos da história da matemática, baseada em tendências historiográficas atuais; 2) para sensibilizar o historiador da matemática envolvido com ensino de matemática a produzir material bibliográfico acessível aos educadores matemáticos (SAITO, 2016, p. 256)

Os diálogos têm a função de incomodar o educador matemático e o historiador de modo que ambos modifiquem suas concepções. Produzindo assim 
dois movimentos. O primeiro movimento faz com que o educador matemático vá ao passado, para compreender como os conceitos matemáticos foram desenvolvidos ou mobilizados na obra estudada, observando os saberes e fazeres da época para a compreensão epistemológica, historiográfica e contextual da matemática apresentada.

Essa compreensão do passado, baseado no passado, leva o educador matemático a investigar o contexto da época, a compreender os fatores sociais, filosóficos, culturais, religiosos e bélicos que influenciaram a elaboração da obra. Durante este processo é buscado a identificação de elementos que poderão ser mobilizados para auxiliar o ensino de um conteúdo matemático, iniciando-se o desenvolver de atividades didáticas que que inclui: a intencionalidade do educador; o plano de ação; o tratamento didático, aplicação da atividade e a obtenção de resultados a partir dessa prática, indicando a construção da referida interface.

\section{Metodologia}

O desenvolvimento deste estudo foi efetivado visando à identificação da integração de duas áreas de conhecimento: a história e a educação matemática nas pesquisas produzidas pelo GPEHM. Para tanto, realizou-se uma pesquisa qualitativa de cunho bibliográfico, a qual "[...] tem como objetivo primordial a descrição das características de determinada população ou fenômeno ou, então, o estabelecimento de relações entre variáveis." (GIL, 2002, p. 42). Vale ressaltar que:

O método qualitativo é adequado aos estudos da história, das representações e crenças, das relações, das percepções e opiniões, ou seja, dos produtos das interpretações que os humanos fazem durante suas vidas, da forma como constroem seus artefatos materiais e a si mesmos, sentem e pensam (MINAYO, 2008, p.57).

Ou seja, buscou-se compreender o fenômeno da construção de interfaces envolvendo instrumentos matemáticos a partir do contexto histórico de sua 
construção e uso, considerando as variáveis implicadas nos trabalhos publicados pelo GPEHM, subsidiadas com embasamento teórico das áreas envolvidas.

O GPEHM foi criado em 2013 na Universidade Estadual do Ceará (UECE), com o objetivo de realizar pesquisas sobre o desenvolvimento teórico de temas ligados a história da matemática para a preparação de estudantes, professores e pesquisadores que irão atuar na área da educação matemática. Visando a coleta de dados, foi realizado um levantamento dos estudos de integrantes do GPEHM que foram divulgados em revistas, anais de eventos e livros, no período de 2016 a 2018, pois corresponde à intensificação do aprofundamento do grupo na temática definida neste trabalho.

Dessa forma, oportunizou-se elencar elementos que estas produções apresentam em consonância com a construção de interface, em que etapa desse processo elas se encontram, indicando os passos a seguir que sinalizam momentos futuros das pesquisas que culminarão no processo completo de interface entre a história e o ensino de matemática para os casos específicos dos instrumentos que elas tratam e os textos históricos que se relacionam com eles.

\section{Estudos realizados no GPEHM que vislumbram o uso da interface e instrumentos matemáticos}

A seguir, apresenta-se as pesquisas realizadas pelo GPEHM no período de 2016 a 2018 no que se refere a estudos voltados a construção de interfaces entre história e ensino e/ou o uso de instrumentos matemáticos, as quais foram identificadas pela busca em livros, periódicos de revistas e anais de eventos. Elenca-se ainda como forma de guiar nossa análise uma possível categorização para tais trabalhos, a qual foi estabelecida a partir de uma leitura em seus resumos.

No Quadro 1, a seguir, expõe-se 19 pesquisas coletadas no período de tempo citado anteriormente, em sua disposição mostra-se o título dos estudos, os autores e o ano de publicação de cada divulgação científica. 


\begin{tabular}{|c|c|c|c|}
\hline & Título do trabalho & Autores & Ano \\
\hline $\mathbf{P 1}$ & $\begin{array}{l}\text { Investigando as potencialidades didáticas dos instrumentos } \\
\text { históricos no ensino de matemática }\end{array}$ & Pereira & 2016 \\
\hline $\mathbf{P 2}$ & $\begin{array}{l}\text { Uma breve discussão teórica acerca do uso de instrumentos } \\
\text { matemáticos históricos no ensino da matemática }\end{array}$ & Batista e Alves & 2016 \\
\hline P3 & $\begin{array}{l}\text { Redescobrindo o conceito de logaritmo por meio da construção da } \\
\text { régua de cálculo linear }\end{array}$ & $\begin{array}{l}\text { Martins, Pereira } \\
\text { e Fonseca }\end{array}$ & 2016 \\
\hline $\mathbf{P 4}$ & $\begin{array}{l}\text { Instrumentos Históricos e o Ensino de Matemática: a Régua de } \\
\text { Cálculo Circular e suas contribuições na formação do professor. }\end{array}$ & Alves e Pereira & 2016 \\
\hline P5 & A matemática do kamal: uma possibilidade de inserção no ensino & $\begin{array}{l}\text { Batista e } \\
\text { Pereira }\end{array}$ & 2016 \\
\hline P6 & $\begin{array}{l}\text { Vamos aprender trigonometria? Uma experiência com alunas no } \\
\text { ensino médio utilizando a balestilha }\end{array}$ & $\begin{array}{l}\text { Batista e } \\
\text { Pereira }\end{array}$ & 2016 \\
\hline P7 & $\begin{array}{l}\text { Algumas potencialidades didáticas da Balestilha na formação de } \\
\text { professores. }\end{array}$ & Batista & 2016 \\
\hline P8 & $\begin{array}{l}\mathrm{O} \text { ensino de conceitos geométricos e trigonométricos por meio } \\
\text { da balestilha: uma experiência na formação inicial de professores. }\end{array}$ & $\begin{array}{l}\text { Batista e } \\
\text { Pereira }\end{array}$ & 2016 \\
\hline P9 & $\begin{array}{l}\text { A inserção da régua de cálculo circular como ferramenta para o } \\
\text { ensino de logaritmo }\end{array}$ & $\begin{array}{l}\text { Alves, Silva, } \\
\text { Pereira }\end{array}$ & 2017 \\
\hline $\mathbf{P 1 0}$ & $\begin{array}{l}\text { A balestilha descrita na obra chronographia repertorio dos tempos: } \\
\text { discussões iniciais sobre o saber incorporado no instrumento }\end{array}$ & $\begin{array}{l}\text { Pereira, Batista } \\
\quad \text { e Silva }\end{array}$ & 2017 \\
\hline P11 & $\begin{array}{l}\text { A matemática incorporada na construção do quadrante descrito na } \\
\text { obra Libros del Saber de Astronomia. }\end{array}$ & $\begin{array}{l}\text { Pereira, Batista } \\
\text { e Silva }\end{array}$ & 2017 \\
\hline $\mathbf{P 1 2}$ & $\begin{array}{l}\text { A evolução histórica da multiplicação no século XVI: Construindo } \\
\text { interfaces para o Ensino }\end{array}$ & $\begin{array}{l}\text { Pereira, Martins } \\
\quad \text { e Silva }\end{array}$ & 2017 \\
\hline $\mathbf{P 1 3}$ & $\begin{array}{l}\text { Articulando conceitos matemáticos e história da matemática: } \\
\text { a balestilha na interface }\end{array}$ & $\begin{array}{l}\text { Batista e } \\
\text { Pereira }\end{array}$ & 2017 \\
\hline P14 & $\begin{array}{l}\text { Compreendendo alguns conceitos matemáticos por meio } \\
\text { da balestilha como recurso didático: um olhar dos licenciandos em } \\
\text { matemática }\end{array}$ & $\begin{array}{l}\text { Batista, Silva e } \\
\quad \text { Pereira }\end{array}$ & 2017 \\
\hline P15 & $\begin{array}{l}\text { A balestilha: um instrumento náutico como recurso para abordar } \\
\text { conceitos matemáticos }\end{array}$ & $\begin{array}{l}\text { Batista e } \\
\text { Pereira }\end{array}$ & 2017 \\
\hline P16 & $\begin{array}{l}\text { O Ensino de Aritmética por Meio de Instrumentos: uma Abordagem } \\
\text { utilizando a obra Rabdologiae, seu numerationis per virgula }\end{array}$ & $\begin{array}{l}\text { Pereira e } \\
\text { Martins }\end{array}$ & 2017 \\
\hline $\mathbf{P 1 7}$ & $\begin{array}{l}\text { O uso da balestilha para articular história da matemática e conceitos } \\
\text { matemáticos: a construção de uma interface }\end{array}$ & $\begin{array}{l}\text { Batista e } \\
\text { Pereira }\end{array}$ & 2017 \\
\hline P18 & $\begin{array}{l}\text { A matemática por trás da construção física e graduação da régua de } \\
\text { cálculo circular }\end{array}$ & Alves, Pereira & 2018 \\
\hline P19 & $\begin{array}{l}\text { Estudos iniciais sobre o instrumento cubit rod: teoria e prática na } \\
\text { história da matemática }\end{array}$ & $\begin{array}{l}\text { Silva, Batista, } \\
\text { Pereira }\end{array}$ & 2018 \\
\hline
\end{tabular}

Quadro 1 - Pesquisas que vem sendo desenvolvidas no GPEHM sobre a construção de uma interface entre história e ensino e/ou o uso de instrumentos.

Fonte: Elaborado pelos autores 
Os trabalhos citados no Quadro 1, em sua maioria, foram publicados como artigos em periódicos de revista (12 trabalhos), 4 em anais de eventos, 2 como livros completos e um como capítulo de livro. Assim, entende-se que tais estudos apresentam certo grau de "impacto" e/ou de "inovação" frente a abordagens de incorporação de elementos da história da matemática no ensino, pois as revistas em sua maioria valorizam tais características em uma pesquisa.

Para efeito de análise, os trabalhos foram agrupados no quadro 2, conforme as semelhanças de suas aplicações. Estes grupos tem a finalidade de facilitar a identificação de quais etapas da construção de interfaces foram alcançadas durante o desenvolvimento dos trabalhos desenvolvidos no GPEHM, com a intenção de elencar esses elementos.

\begin{tabular}{|l|l|l|}
\hline & \multicolumn{1}{|c|}{ Categorias de análise } & \multicolumn{1}{c|}{ Pesquisas } \\
\hline C1 & Ensaios teóricos & $\mathrm{P} 1$ e P2 \\
\hline C2 & $\begin{array}{l}\text { Instrumentos matemáticos usados em } \\
\text { Cálculo numérico ou de medidas }\end{array}$ & $\mathrm{P} 3, \mathrm{P} 4, \mathrm{P} 9, \mathrm{P} 12, \mathrm{P} 16, \mathrm{P} 18$ e P19 \\
\hline C3 & $\begin{array}{l}\text { Instrumentos Náuticos e } \\
\text { Astronômicos }\end{array}$ & $\begin{array}{l}\mathrm{P} 5, \mathrm{P} 6, \mathrm{P} 7, \mathrm{P} 8, \mathrm{P} 10, \mathrm{P} 11, \mathrm{P} 13, \\
\mathrm{P} 14, \mathrm{P} 15 \text { e P17 }\end{array}$ \\
\hline
\end{tabular}

Quadro 2 - Categorias estabelecidas para análise das pesquisas

Fonte: Elaborado pelos autores

De tais categorias, observa-se que as pesquisas desenvolvidas no GPEHM estão voltadas a atividades em sala de aula, visto que as investigações em sua maioria não se caracterizam como ensaios teóricos. Desenvolvem-se por meio de uma investigação com instrumentos matemáticos, sejam estes para fins de cálculo numérico ou de medidas ou mesmo para atividades voltadas a astronomia ou a náutica com vistas a evidenciar deles elementos a serem incorporados no ensino.

A seguir, expõe-se a análise dos estudos em suas respectivas categorias de acordo com os critérios definidos pelas etapas de construção da interface almejada (contextualização histórica, movimento do pensamento, definição das 
potencialidades didáticas, elaboração e aplicação de atividades investigativas e resultados), bem como o tipo de historiografia adotada (tradicional ou atualizada).

\subsection{Ensaios teóricos}

Os estudos presentes nos trabalhos P1 e P2, de Pereira (2016) e Batista e Alves (2016) fazem ensaios teóricos sobre o uso de instrumentos históricos, perpassando pelas potencialidades didáticas que podem emergir desses materiais históricos até o uso destes elementos em sala de aula e a elaboração de atividades didáticas.

No entanto, por se tratarem de estudos teóricos, não trazem a aplicação das atividades elaboradas nem a contextualização histórica em torno de algum instrumento específico. Indicando que o intuito destes não consiste em construir interface entre a história e o ensino, mas em fortalecer as bases teóricas para as pesquisas que estavam em andamento.

\subsection{Instrumentos matemáticos usados em Cálculo}

Com relação aos instrumentos em si, podem-se encontrar três pesquisas sobre a Régua de Cálculo Circular (P4, P9 e P18). O trabalho P4 de Alves e Pereira (2016) foi produzido a partir da aplicação de um minicurso na formação de professores de matemática, no qual foi explorada a utilização da Régua de Cálculo Circular a partir da Obra The Circles of Proportion, de Willian Oughtred, não havendo uma contextualização histórica ou historiográfica a partir do escrito referência.

Indicando que não houve a construção de interfaces por se tratar ainda de uma abordagem historiográfica tradicional que "preocupa-se com problemas internos à matemática, independentemente do contexto social ou do meio em que eram produzidos" (SAITO, 2015, p. 24).

Já no trabalho P9 das autoras Alves, Silva e Pereira (2016), a régua de cálculo é mencionada como um recurso didático e são direcionadas orientações para a construção e uso com relação ao conteúdo de logaritmos, a partir da 
matemática mobilizada no século XVII, fato que preparou caminho para a aplicação dessa construção e uso, emergindo saberes matemáticos e indicando possíveis potencialidades didáticas destes elementos históricos, esboçando a intenção da construção de uma interface.

Enquanto o artigo P18, Alves e Pereira (2018) dão ênfase a construção física e a graduação da régua de cálculo circular, com uma breve contextualização histórica do instrumento a partir de elementos da obra histórica "The Circles of Proportion and the Horizontal Instrument", do matemático inglês Willian Oughtred, no ano de 1633. Foi apresentada ainda a dimensão epistemológica dos conceitos matemáticos envolvidos, de acordo com os conhecimentos do passado. A partir daí foi sugerida a elaboração de uma atividade para futuras aplicações no ensino.

A pesquisa P3 de Martins, Pereira e Fonseca (2016) com régua de cálculo, baseia-se na compreensão histórica da construção da régua de cálculo, de modo a identificar os conceitos matemáticos que estavam disponíveis na época e que foram mobilizados, possibilitando a construção do instrumento de modo que sua utilização respeite às normas operacionais vigentes no passado.

Com relação aos trabalhos P16 de Pereira, Martins (2017) e P12 de Pereira, Martins e Silva (2017), eles apresentam o contexto histórico das diferentes formas de realizar a operação de multiplicação que encontram se presente em obras que introduziram na Europa os algarismos indo-arábicos e como operar com eles, do século $\mathrm{X}$ ao $\mathrm{XVII}$ e finalizando com um instrumento construído para a realização de cálculos aritméticos. Estes estudos realizam 0 primeiro movimento analisando o contexto histórico e epistemológico da época. Porém, os autores não se prendem apenas a estes contextos e fazem um rápido ensaio de aplicação dos conteúdos estudados para o ensino.

\subsection{Instrumentos Náuticos e Astronômicos}

No que se refere às pesquisas desenvolvidas tendo a balestilha como instrumento matemático a ser possivelmente incorporado em sala, listadas na 
tabela anterior, observa-se nos trabalhos P6, P8, P14 e P15, uma abordagem do instrumento histórico sob uma perspectiva historiográfica tradicional. Nos demais, ou seja, os P7, P10, P13 e P17, nota-se a presença de elementos atreladas a noção de construção de interface entre história e ensino.

Tal observação quanto a proximidade dos estudos de Batista e Pereira (2016a), Batista e Pereira (2016b), Batista, Silva e Pereira (2017) e Batista e Pereira (2017b) com uma perspectiva de trabalho da história sob uma abordagem historiográfica tradicional, se fundamenta a partir das considerações teóricas de Saito (2015). Posto que a forma como o instrumento foi estudado e incorporado no ensino não comtempla características de uma vertente historiográfica atualizada.

Em tais estudos a balestilha, instrumento utilizado na navegação principalmente nos séculos XVI e XVII é investigada a partir de conhecimentos que hoje nos são familiar, observa-se por meio das atividades nelas realizadas quanto a construção e/ou uso da balestilha uma tentativa de extrair da história elementos que favorecessem o ensino de matemática.

Por sua vez, tem-se que as pesquisas de Batista (2016), Pereira, Batista e Silva (2017), Batista e Pereira (2017a) e a de Batista e Pereira (2017c) já incorporam de certa forma elementos de uma historiografia atualizada, pois se observou dentre outros aspectos, a tentativa de investigar o instrumento levando em considerações não apenas questões internas a própria matemática, mas também históricas e contextuais.

Destas tentativas, cabe desatacar a de Batista e Pereira (2017a), em que buscam apresentar uma proposta de trabalho com a balestilha a ser desenvolvida sob a perspectiva da vertente historiográfica atualizada. Nela observa-se que as autoras vislumbram construir uma interface entre história e ensino da matemática, prova disso é que buscam iniciar uma articulação do objeto da pesquisa com as esferas epistemológica, historiográfica e contextual de análise.

Outro exemplo, dessa similaridade, repousa no estudo de Pereira, Batista e Silva (2017) em que buscam apresentar a Balestilha por meio de uma discussão 
entre o saber e o fazer incorporado nela verificados a partir das considerações a seu respeito descritas na obra Chronographia repertorio dos tempos, escrita em 1603. Dessa intenção, entende-se que está de certa forma atrelada aos estudos iniciais que permitem o estabelecimento da construção de interface, os quais devem revelar questões historiográficas, epistemológicas (contexto onde as ideias matemáticas estão mobilizadas).

Das pesquisas sobre a balhestilha derivou o conhecimento do Kamal como instrumento matemático do século $\mathrm{XV}$, que se trata de uma espécie de balhestilha confeccionada com uma tábua e um cordão com amarrações. Pereira e Batista (2016) revelam o contexto histórico em torno desse instrumento e incluem a matemática envolvida nesse processo, gerando possibilidades de uso voltado para o ambiente escolar. Houve aplicação desses conhecimentos na formação de professores como meio de integrar a teoria com a prática. No entanto, o uso de textos originais não foi identificado, mas sim fontes secundárias.

Em linhas gerais, Pereira, Batista e Silva (2017) (P11) trazem um estudo sobre a construção do instrumento Quadrante que ocorreu a partir de informações contidas na obra Libros del Saber de Astronomía. Foi estruturado com fundamentos da educação matemática e a questão dos instrumentos históricos para o ensino. As autoras trouxeram a história envolvida com esse instrumento e demonstraram os passos propostos para a construção, por meio de construções geométricas, dedicando a trabalhos futuros a utilização deste instrumento.

\section{Considerações finais}

No presente trabalho, elucidamos alguns elementos que têm sido abordados em estudos que tencionam construir uma interface entre história e ensino da matemática, destes, pode-se observar a importância que dos instrumentos matemáticos investigados a partir de um documento histórico que tem para poder reconstruir conceitos da malha histórica neles incorporados.

O enfoque historiográfico tradicional é sinalizado nos trabalhos, mas pela brevidade dos mesmos, possui recortes bem definidos, o que demonstra que 
"toda narrativa da história da matemática é historicamente orientada. Isso significa que as narrativas históricas não são neutras e são influenciadas por diferentes fatores ligados à concepção de ciência (isto é, de conhecimento válido numa época)" (SAITO, 2015, p. 21).

Assim, os educadores matemáticos que trabalham com os instrumentos históricos como recursos didáticos, adotando a perspectiva historiográfica atualizada, delimitam um campo de atuação a ser desbravado, com fundamentação teórica e potencial para que a ação seja na formação de professores ou diretamente na sala de aula com os estudantes nos mais diversos níveis de ensino.

Outra característica de tais estudos é que elas têm como foco chegar a execução de ações em sala de aula seja na educação básica ou nos cursos de formação de professores. Tal objetivo indica que a proposta de construção de interface está sendo formalizada na concepção dos pesquisadores, já que o estudo histórico é realizado não como fim, mas como meio de visualizar os conhecimentos a serem incorporados no ensino.

Portanto, a partir das produções do GPEHM no período de 2016 a 2018 foi possível estabelecer o perfil das pesquisas que estão sendo desenvolvidas sobre interface por este coletivo, de maneira que os elementos da construção de interface se tornaram perceptíveis, tendo em vista ainda resquícios de historiografias tradicionais ou até mesmo a não realização das etapas completas da interface que contemplam o contexto onde as ideias matemáticas estão mobilizadas, o movimento do pensamento de onde emergem as potencialidades didáticas, a elaboração e aplicação de atividades e os resultados desse processo.

Ademais, diante das informações elencadas, entende-se que estudos votados à construção de interface entre história e ensino indicam um caminho a se pensar, estudar, trabalhar elementos da história da matemática no ensino e não os sobrepor aos temas e propósitos já existentes. 


\section{Referências}

ALVES, Verusca Batista; PEREIRA, Ana Carolina Costa. A matemática por trás da construção física e graduação da régua de cálculo circular. Caminhos da Educação Matemática em Revista/online, Sergipe, v. 8, n. 2, p.11-30, 2018. Disponível em: $<$ https://aplicacoes.ifs.edu.br/periodicos/index.php/caminhos da educacao matematica/a rticle/view/191/162>. Acesso em: 09 maio 2018.

ALVES, Verusca Batista; PEREIRA, Ana Carolina Costa. Instrumentos históricos e o ensino de Matemática: a Régua de Cálculo Circular e suas contribuições na formação do professor. Remat: Revista Eletrônica da Matemática, Caxias do Sul, v. 2, n. 2, p.39-50, 2016. Disponível em:

< https://periodicos.ifrs.edu.br/index.php/REMAT/article/view/1554/1302>. Acesso em: 09 maio 2018.

ALVES, Verusca Batista; BATISTA, Antonia Naiara de Sousa. Uma breve discussão teórica acerca do uso de instrumentos matemáticos históricos no ensino da

matemática. Boletim Cearense de Educação e História da Matemática, Fortaleza, v. 3, n. 8, p.48-59, 2016. Disponível em:

$<$ http://seer.uece.br/?journal=BOCEHM\&page=article\&op=view\&path\%5B\%5D=2030\&pa th\%5B\%5D=184>. Acesso em: 09 maio 2018.

ALVES, Verusca Batista; SILVA, Hosana de Fátima Melo da; PEREIRA, Ana Carolina Costa. A inserção da régua de cálculo circular como ferramenta para o ensino de logaritmo. Boletim Cearense de Educação e História da Matemática, Fortaleza, v. 4, n. 10, p.48-60, 2017. Disponível em:

$<$ http://seer.uece.br/?journal=BOCEHM\&page=article\&op=view\&path\%5B\%5D=2403\&pat

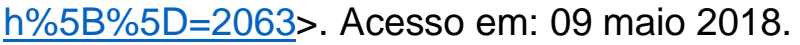

BARONI, Rosa Lucia Sverzut; NOBRE, Sergio Roberto. A Pesquisa em História da Matemática e suas relações com a Educação Matemática. In: Maria Aparecida V. Bicudo. (Org.). Pesquisa em Educação Matemática: Concepções \& Perspectivas. 1ed. São Paulo: Editora Unesp, 1999, v. 1, p. 129-136.

BATISTA, Antonia Naiara de Sousa; PEREIRA, Ana Carolina Costa. A balestilha: um instrumento náutico como recurso para abordar conceitos matemáticos. HipÁtia - Revista Brasileira de História, Educação e Matemática, São Paulo, v. 2, n. 1, p.40-51, 2017b. Disponível em: < http://ojs.ifsp.edu.br/index.php/hipatia/article/view/265/180>. Acesso em: 09 maio 2018.

BATISTA, Antonia Naiara de Sousa; PEREIRA, Ana Carolina Costa. Articulando conceitos matemáticos e história da matemática: a balestilha na interface. In: Congresso internacional de educação cientifica e tecnológica (CIECITEC), 4., 2017, Santo Ângelo -

Rs. Anais do IV Congresso Internacional de Educação Cientifica e

Tecnológica. Santo Ângelo - Rs: Universidade Regional Integrada do Alto Uruguai e das Missões - Uri, 2017a. v. 1, p. 1 - 7. Disponível em:

< http://www. santoangelo.uri.br/anais/ciecitec/2017/home.htm\#>. Acesso em: 09 maio 2018. 
BATISTA, Antonia Naiara de Sousa; PEREIRA, Ana Carolina Costa. O ensino de conceitos geométricos e trigonométricos por meio da balestilha: uma experiência na formação inicial de professores. In: XVI - encontro de pós-graduação e pesquisa, 16., 2016, Fortaleza. Anais do XVI - Encontro de Pós-Graduação e Pesquisa. Fortaleza: Universidade de Fortaleza, 2016b. p. 1 - 6. Disponível em: $<$ http://uol.unifor.br/oul/conteudosite/?cdConteudo=6953133>. Acesso em: 09 maio 2018.

BATISTA, Antonia Naiara de Sousa; PEREIRA, Ana Carolina Costa. O uso da balestilha para articular história da matemática e conceitos matemáticos: a construção de uma interface. In: XVII - encontro de pós-graduação e pesquisa, 17., 2017, Fortaleza. Anais do XVII - Encontro de Pós-Graduação e Pesquisa. Fortaleza: Universidade de Fortaleza, 2017c. p. 1 - 6. Disponível em: $<$ http://uol.unifor.br/oul/conteudosite/?cdConteudo=7890913>. Acesso em: 09 maio 2018.

BATISTA, Antonia Naiara de Sousa; PEREIRA, Ana Carolina Costa. Vamos aprender trigonometria? uma experiência com alunas no ensino médio utilizando

a balestilha. Boletim Cearense de Educação e História da Matemática, Fortaleza, v. 3, n. 7, p.54-65, 2016a. Disponível em:

$<$ http://www.seer.uece.br/?journal=BOCEHM\&page=article\&op=view\&path\%5B\%5D=158 5\&path\%5B\%5D=1382>. Acesso em: 09 maio 2018.

BATISTA, Antonia Naiara de Sousa; SILVA, Isabelle Coelho da; PEREIRA, Ana Carolina Costa. Compreendendo alguns conceitos matemáticos por meio da balestilha como recurso didático: um olhar dos licenciandos em matemática. In: Congresso internacional de educação cientifica e tecnológica (CIECITEC), 4., 2017, Santo Ângelo - Rs. Anais do IV Congresso Internacional de Educação Cientifica e Tecnológica. Santo Ângelo -

Rs: Universidade Regional Integrada do Alto Uruguai e das Missões - Uri, 2017. v. 1, p. 1 - 10. Disponível em: < http://www.santoangelo.uri.br/anais/ciecitec/2017/home.htm\#>. Acesso em: 09 maio 2018.

GIL, Antônio Carlos. Como elaborar projetos de pesquisa. 4. ed. São Paulo: Atlas, 2002.

KILPATRICK, Jeremy. Investigación em educación matemática: su história y algunos temas de actualidad. In: KILPATRICK, J. GÓMEZ, P. RICO, L. Errores y dificuldade de los estudiantes. Bogotá: Universidade de los Andes, 1998.

LORENZATO, Sérgio e FIORENTINI, Dario. O profissional em Educação Matemática. Santos: UNISANTA, 2001.

MARTINS, Eugeniano Brito; PEREIRA, Ana Carolina Costa; FONSECA, Paulo Henrique Souza. Redescobrindo o conceito de logaritmo por meio da construção da régua de cálculo linear. Revista Eletrônica Debates em Educação Científica e Tecnológica, Espírito Santo, v. 6, n. 3, p.47-65, 2016. Disponível em:

< http://ojs.ifes.edu.br/index.php/dect/article/view/498>. Acesso em: 09 maio 2018. 
MINAYO, Maria Cecília de Souza. O desafio do conhecimento. 11 ed. São Paulo: Hucitec, 2008

PEREIRA, Ana Carolina Costa; BATISTA, Antonia Naiara de Sousa. Algumas potencialidades didáticas da balestilha na formação de professores. In: PEREIRA, Ana Carolina Costa; ALVES, Francisco Regis Vieira; VASCONCELOS, Francisco Herbert Lima. Ensino de ciências e matemática: enfoques de práticas docentes. Fortaleza: Imprima, 2016. p. 187-205.

PEREIRA, Ana Carolina Costa; BATISTA, Antônia Naiara de Sousa. A matemática do kamal: uma possibilidade de inserção no ensino. Conexões - Ciência e Tecnologia, Fortaleza, v. 10, n. 4, p.25-34, 2016. Disponível em:

$<$ http://conexoes.ifce.edu.br/index.php/conexoes/article/view/1141/834>. Acesso em: 09 maio 2018.

PEREIRA, Ana Carolina Costa; BATISTA, Antônia Naiara de Sousa; SILVA, Isabelle Coelho da. A balestilha descrita na obra chronographia repertorio dos tempos (1603): discussões iniciais sobre o saber incorporado no instrumento. Boletim Cearense de Educação e História da Matemática, Fortaleza, v. 4, n. 11, p.105-108, 2017. Disponível em:

$<$ http://seer.uece.br/?journal=BOCEHM\&page=article\&op=view\&path\%5B\%5D=2644\&pat h\%5B\%5D=2249>. Acesso em: 09 maio 2018.

PEREIRA, Ana Carolina Costa; BATISTA, Antônia Naiara de Sousa; SILVA, Isabelle Coelho da. A matemática incorporada na construção do quadrante descrito na obra Libros del Saber de Astronomía. Revista Eletrônica de Educação Matemática, Florianópolis, v. 12, n. 1, p.173-191, 2017. Disponível em:

$<$ https://periodicos.ufsc.br/index.php/revemat/article/view/19811322.2017v12n1p173/349 32>. Acesso em: 09 maio 2018.

PEREIRA, Ana Carolina Costa. Investigando as potencialidades didáticas dos instrumentos históricos no ensino de matemática. In: Seminário Nacional de História da Ciência e da Tecnologia, 15, 2016, Florianópolis. Anais... . Rio de Janeiro: Sociedade Brasileira de História da Ciência, 2016. p. 1 - 16.

PEREIRA, Ana Carolina Costa; MARTINS, Eugeniano Brito. O Ensino de Aritmética por Meio de Instrumentos: uma Abordagem utilizando a obra Rabdologiae, seu numerationis per virgula. São Paulo: Editora da Física, 2017.

PEREIRA, Ana Carolina Costa; MARTINS, Eugeniano Brito; SILVA, Isabelle Coelho da. A EVOLUÇÃOO HISTÓRICA DA MULTIPLICAÇÃ̃O DO SÉCULO X AO XVI: Construindo interfaces para o Ensino. Belém: Sbem-pa, 2017.

SAITO, Fumikazu; DIAS, Marisa da Silva. Interface entre história da matemática e ensino: uma atividade desenvolvida com base num documento do século XVI. Ciência \&

Educação. v. 19, n. 1. p. 89-111, 2013.

SAITO, Fumikazu. História da matemática e suas (re)construções contextuais. São Paulo: Livraria da Física, 2015. 
SAITO, Fumikazu. História e Ensino da matemática: Construindo Interfaces. In: SALAZAR, Jesús Flores e GUERRA, Francisco Ugarte (eds). Investigaciones em Educacion Matemática. Lima: Fondo Editorial PUCP, 2016, p. 253-291.

SILVA, Isabelle Coelho da; BATISTA, Antonia Naiara de Sousa; PEREIRA, Ana Carolina Costa. Estudos iniciais sobre o instrumento cubit rod: teoria e prática na história da matemática. Caminhos da Educação Matemática em Revista/online, Sergipe, v. 8, n. 2, p.1-10, maio 2018. Disponível em:

$<$ https://aplicacoes.ifs.edu.br/periodicos/index.php/caminhos da educacao matematica/a rticle/view/189/160>. Acesso em: 09 maio 2018. 